\title{
Response to: A Telehealth Explosion: Using Lessons From the Pandemic To Shape the Future of Telehealth Regulation
}

Joanna Sax

California Western School of Law, jsax@cwsl.ed

Follow this and additional works at: https://scholarship.law.tamu.edu/lawreview

Part of the Telemedicine Commons

\section{Recommended Citation}

Joanna Sax, Response to: A Telehealth Explosion: Using Lessons From the Pandemic To Shape the Future of Telehealth Regulation, 9 Tex. A\&M L. Rev. Arguendo 25 (2021).

Available at: https://doi.org/10.37419/LR.V9.Arg.3

This Arguendo (Online) is brought to you for free and open access by Texas A\&M Law Scholarship. It has been accepted for inclusion in Texas A\&M Law Review by an authorized editor of Texas A\&M Law Scholarship. For more information, please contact aretteen@law.tamu.edu. 


\title{
Response to: A Telehealth Explosion: Using Lessons From the Pandemic To Shape THE FUTURE OF TELEHEALTH REGULATION
}

\author{
by: Joanna K. Sax, J.D. Ph.D. *
}

In A Telehealth Explosion: Using Lessons from the Pandemic to Shape the Future of Telehealth Regulation, published in the Texas A\&M Law Review, Professor Deborah Farringer tackles the critical issue of the efficacy and implementation of telehealth, using our experience(s) of telehealth during the COVID-19 pandemic as the guide. This is important, as Professor Farringer acknowledges, because while telehealth advocates pre-date the pandemic, barriers prevented the implementation of telehealth in a widespread manner. These barriers included a concern about fraud and a question as to whether telehealth visits could provide effective outcomes compared to in-person visits. Professor Farringer reflects on these barriers and addresses their validity, especially given what we learned through the COVID-19 pandemic. Professor Farringer then moves the discussion forward to propose that not only can our healthcare system utilize telehealth, but it can do so in a more meaningful way. This can be done by addressing the benefits and risks we learned through the waivers that allowed telehealth visits during the COVID-19 pandemic.

The context for the article is the utilization of telehealth during the COVID-19 pandemic. Telehealth was important to reduce the spread of the virus that causes COVID-19. The medical concern was bringing patients into offices and hospitals, where the virus could be transmitted. The solution to this was video visits with doctors. The emergency context of the COVID-19

DOI: https://doi.org/10.37419/LR.V9.Arg.3

* Joanna K. Sax is the E. Donald Shapiro Professor of Law at California Western School of Law in San Diego, CA. Professor Sax is grateful to the editorial board of the Texas A\&M Law Review for the invitation to publish a response to Professor Farringer's excellent article. 
pandemic, and the reasons telehealth exploded during the COVID-19 pandemic, allow for reflection on why telehealth access has been limited. As Professor Farringer notes, telehealth "was neither new nor novel at the time of the coronavirus pandemic in 2020." As we move out of this COVID-19 pandemic, eventually, why not continue telehealth mechanisms, even if we are not practicing pandemic-non-pharmaceutical interventions? Telehealth, this oddly controversial area of medical delivery, should not have such a strange place in our healthcare system. Professor Farringer uses the experience of telehealth during the COVID-19 pandemic to offer insight as to how this can be more mainstream. She aptly refers to the COVID-19 pandemic as the "tipping point" to explore an expansion of telehealth services in a post-pandemic world.

Professor Farringer defines what is meant by telehealth as the use of technology and telemedicine to render clinical services. Within this broad definition are four categories: (1) mobile health (mHealth); (2) video and audio technology; (3) remote patient monitoring (RPM); and (4) store-and-forward technologies. Professor Farringer's article addresses telehealth broadly, although each of these four categories has its own nuance.

In Part II, Professor Farringer discusses the historical barriers to telehealth, including licensing and reimbursement. State licensing has always been a barrier to entry with the stated purpose that licensing is a quality control measure. While this may be true, it is unclear why state licensing, as opposed to a national licensing program, could not effectuate the same purpose. The state licensing serves as a barrier because physicians cannot practice across state lines unless they are part of the Interstate Medical Licensure Compact, which has set criteria for physicians to participate. Another barrier is the requirement, or in some cases limitations, of face-to-face visits to establish a physician-patient relationship to prescribe medications. This limitation is tied to reimbursement rules, which create their own set of barriers as well. One example of the 
reimbursement barriers is the reimbursement for the practitioner and a separate reimbursement for the originating site. The low reimbursement rates for the distant practitioners simply made it so that many specialists chose not to participate. Changes in the Medicare reimbursement system are compounded by findings from the Office of the Inspector General that many claims did not meet the Medicare billing requirements for telehealth services. For Medicaid, states determine the reimbursement rules. As a program largely implemented by the states, it is not surprising to find a patchwork of systems often unable to coordinate with each other. Private insurance poses its own set of reimbursement issues, including parity of reimbursement and other types of restrictions. This Part nicely explains the historical barriers, much of which can be attributed to issues that impact other areas of our healthcare system. In other words, licensing and reimbursement drive much of our healthcare system rather than respond to society's healthcare needs.

Part III describes the waivers to these barriers implemented during the COVID-19 pandemic, which allows for reflection on the utility of the previous barriers. As noted above, these waivers were needed to provide care so that non-pharmaceutical interventions could be followed. This was complicated, to say the least. The Office for Civil Rights governs the security issues related to patient privacy; these rules needed to be relaxed to allow greater flexibility in the types of software applications used for telehealth services. The Centers for Medicare and Medicaid Services (CMS) for Medicare had to waive many limitations on the delivery of telehealth services, including the originating sites requirements, differing reimbursement rates, and intra-state licensing requirements. The Drug Enforcement Agency implemented waivers to adjust prescribing prohibitions and practices. CMS for Medicaid did not appear to waive any requirements, per se, given that the states run Medicaid, but the states instituted waivers similar 
to those found for Medicare recipients. The CMS for Medicaid waivers are federal; thus, it is possible that even with federal waivers, the states may still prohibit some of the activities.

Part III also addresses the state level. At the state level, most states followed suit and made necessary changes. One change was state licensing requirements, which allowed physicians to provide services across state lines. Some states also waived the requirement for an in-person visit before obtaining a prescription. Some states allowed the informed consent to a telehealth visit to be verbal. Professor Farringer also cataloged a series of state laws related to payment parity, increased access to behavioral health services, removal of requirements for inperson visits to set up a physician-patient relationship, and added services available via telehealth. Overall, states changed their rules to allow for the increased use of telehealth. In addition, this article describes calls by legislators and professional organizations to enact and extend the types of waivers and policies related to telehealth that we experienced during the COVID-19 pandemic.

In Part IV, rather than piecemeal the way through the waivers and calls by legislators and professional organizations to determine which policies are effective, Professor Farringer advocates for a broader approach so that telehealth can "thrive" in the future. This approach is advantageous because it creates a framework that allows for flexibility.

The first part of the framework is the coordination and cooperation of state and federal regulators. This suggestion may seem obvious, but it is not. This lack of coordination and cooperation plagues many sectors of healthcare delivery; thus, the promotion of it as a principle is and should be a priority. As Professor Farringer explains, a federal relaxation or waiver of the reimbursement rule to allow patients to receive telehealth services from an out-of-state physician is meaningless if the state itself does not allow an out-of-state physician to practice in their state. 
Professor Farringer promotes a solution to this coordination problem: the federal government can create incentives to encourage coordination. Incentives certainly could work, although it requires quite a bit of action at the state level and states to work together — an added layer of complexity. Another solution could be implementing federal regulations that require coordination for participation in Medicaid. The Medicaid system is a federal statute that states must comply with to carry out the state's Medicaid program. The Medicaid Act has been amended many times since its inception. ${ }^{1}$ Although Medicaid is just one program, if states have to coordinate their telehealth services for Medicaid, it may bleed into private insurance regulations. In addition, the federal government has the carrot and stick option to amend the Affordable Care Act to require or incentivize coordination of care. Given the current political climate, these other suggestions are most likely to be challenged in court, but that is not a reason not to attempt reform in this way.

The second part of the proposed framework reduces geographic and locality barriers. The internet is not local; thus, these traditional locality rules where the patient and physician are in the same location simply should not apply. This rule has been in place for a long time, not just for licensing reasons but to protect against fraud. Medicare required that patients engaging in telehealth services be present at an originating site facility to reduce the risk of fraud. This requirement seems like an archaic way to prevent fraud. Professor Farringer's proposal, which is practical and sensible, requires a much bigger commitment to increasing access to broadband services. This problem, lack of access to broadband, is discussed earlier in the article but warrants additional attention here. If one goal of the telehealth framework is to increase access to

\footnotetext{
${ }^{1}$ Nat'l Fed'n of Indep. Bus. v. Sebelius, 567 U.S. 519, 625-26 (2012) (Ginsberg, J., dissenting) (“Given past expansions, plus express statutory warning that Congress may change the requirements participating States must meet, there can be no tenable claim that the ACA fails for lack of notice.").
} 
medical care, then the disparities in access to broadband internet need to be considered as part of this proposal. By analogy, the closing of K-12 schools during the pandemic highlighted the lack of broadband access. ${ }^{2}$ Online school was meaningless if a student could not get online. Similarly, larger investment into broadband appears to be a necessary variable to fully realize the benefits of this portion of the framework. As Professor Farringer notes, originating sites are reluctant to invest in technology because of low reimbursement rates. This point is well-taken, but originating sites provide access to broadband. A policy goal needs to address broadband in both originating sites and our communities.

The third part of the framework is reconsidering fraud, waste, and abuse controls. As the author notes, we now have months, maybe over a year, of billing data for telehealth visits. We can study whether increased access to telehealth from a person's home impacted fraudulent practices in any meaningful way. Our fraud and abuse laws regulate the providers, not the patients. This portion of the framework discusses that some of the barriers to pre-pandemic telehealth are related to highly restricted regulations, including that the patient is located at an originating site to obtain telehealth services. The purpose of the originating site is to reduce patient fraud. But our fraud and abuse laws are focused on the providers and their billing, prescribing, and referring practices. The query for this portion of the framework is what changes are needed in the current fraud and abuse laws to address concerns with the provider side. If data exists about increased fraud on the patient side, these areas need to be addressed as well, but through separate mechanisms. One area for potential patient-side fraud and abuse is gaming the system to obtain prescriptions for opioids. Telehealth, along with online pharmacies, creates a

\footnotetext{
${ }^{2}$ Lauren Camera, Coronavirus School Closings Expose Digital Divide, U.S. NEws (Mar. 10, 2020, 5:02 PM), https://www.usnews.com/news/education-news/articles/2020-03-10/coronavirus-school-closings-expose-digitaldivide [https://perma.cc/R6FW-AWXA].
} 
potential atmosphere for inappropriate access to opioids. At the same time, barriers related to telehealth also preclude access to treatment for substance-abuse disorders. ${ }^{3}$ The push-pull of the opioid epidemic, especially regarding telehealth services, creates avenues for policymakers to consider how to handle this complex issue.

The fourth part of the framework is a policy choice to promote efficiency and quality of care. In other words, are the patients receiving the same or higher quality of care via telehealth visits compared to in-person visits? Again, as the author points out, we should have data on this. One possible result is that more care was accessed due to the ease of telehealth visits, and this may have reduced hospital admissions. The article correctly focuses on licensing as a barrier because of the problem of physicians providing healthcare across state lines via telehealth. Licensing laws have a complicated story. Due to the market inefficiencies, licensing laws serve to address information asymmetries; thus, in that sense, licensing laws are not controversial. But in other contexts, licensing laws serve as a barrier to access to care. A well-known example of the licensing barrier is the controversy regarding Certified Registered Nurse Anesthetists (CRNAs) to perform certain procedures that the nurses consider within their scope of practice. ${ }^{4}$ But, in this article, Professor Farringer notes that the barrier here is to reduce competition by not allowing healthcare providers licensed in one state to provide medical advice to a patient in another state. In this context, licensing becomes anti-competitive and is unrelated to the quality

\footnotetext{
${ }^{3}$ George \#covidlawbriefing. Substance Use Disorder and Mental Health, The Week in Health Law, PodBEAn, (Mar. 4, 2021), https://twihl.podbean.com/e/231-george-covidlawbriefing-substance-use-disorder-and-mental-health/ [https://perma.cc/39UC-Y59F].

${ }^{4}$ Letter on Proposed Rule on the Proposed Regulation of Interventional Pain Management Services Issued by the Alabama State Board of Medical Examiners, FED. TRADE COMM'N (Nov. 3, 2010), https://www.ftc.gov/sites/default/files/documents/advocacy_documents/ftc-staff-comment-alabama-state-boardmedical-examiners-concerning-proposed-regulation/101109alabamabrdme.pdf [https://perma.cc/VG97-T5HA]; see also Nicole Huberfeld, Elizabeth Weeks Leonard \& Kevin Outterson, THE LAW OF AMERICAN HeAlTH CARE 44345 (Wolters Kluwer, 2nd ed. 2018).
} 
of care. For this reason, these barriers should be reduced while still maintaining the quality of care.

The fifth part of the framework is promoting and enhancing reimbursement and payment parity. Reimbursement is a potential barrier to telehealth if the current reimbursement rates for telehealth visits remain below the reimbursement rates for in-person visits. If the quality of care is the same, the reimbursement should be the same. Furthermore, telehealth services require investments into infrastructure - an investment that cannot be made if reimbursements are not in parity with in-person care. The infrastructure investment should arguably be funded separately from reimbursement rules. Again, access to broadband is a larger issue than just telehealth. Even within a medical office, it seems that grants or incentives can be provided for actual investment in technology, especially for those offices that serve Medicaid patients. Because reimbursement rates for Medicaid recipients are usually lower than those with Medicare or private insurance, additional measures are needed for those offices that serve the neediest among us. Additional measures will likely require investment beyond just parity of reimbursement rates.

The author concludes with words of optimism and caution. According to Professor Farringer, adopting the waivers that occurred during the pandemic would be a mistake. Instead, we should learn from and reflect on what went well and what did not during the COVID-19 pandemic to have an intentional telehealth system: one that can be guided by data and principles.

The framework laid out by the article is both practical and compelling. Perhaps it applies to all the four categories of telehealth defined earlier in the article, but it appears to be most relevant to category two: video and audio technology.

Perhaps too far a leap, but one of the issues not discussed in this article is the prohibition of telehealth in clinical settings that clearly should fall within the waivers utilized during the 
COVID-19 pandemic or the framework laid out in this article. For example, during the COVID19 pandemic, Texas Governor Gregg Abbott prohibited abortion procedures, deeming them medically unnecessary. ${ }^{5}$ Setting aside that abortion is not a medically unnecessary procedure, telehealth offers an attractive alternative, especially if the woman can use an abortifacient. ${ }^{6}$ No clinical reason exists for an in-person visit for an otherwise healthy woman who learns of a pregnancy early on and decides to terminate. The abortifacient is safe and effective. Doctors can prescribe via a telehealth visit. How does the framework expressed in this article tackle this issue?

Another important issue, that telehealth offers some opportunity to solve, is compliance with medical advice, such as taking prescription medicine on time. Telehealth, broadly defined, provides an opportunity for mobile reminders, check-ins, self-monitoring, and other types of nudges. ${ }^{7}$ Privacy concerns certainly exist. What if the individual loses their mobile device? How does the framework address the benefits versus the risks? How does reimbursement work for the monitoring of the patients through these types of telehealth measures? This reimbursement issue likely falls under category three: remote patient monitoring. The framework, as proposed, does not squarely address this category.

\footnotetext{
${ }^{5}$ Ashley Lopez, Texas Officials Say Abortions Are Included in Abbott's Order Barring Unnecessary Medical Procedures, KUT 90.5 (Mar. 23, 2020, 4:04 PM), https://www.kut.org/health/2020-03-23/texas-officials-sayabortions-are-included-in-abbotts-order-barring-unnecessary-medical-procedures [https://perma.cc/N3NW-364G]; Tex. Exec. Order No. GA-09 (Mar. 22, 2020), https://lrl.texas.gov/scanned/govdocs/Greg\%20Abbott/2020/GA09.pdf [https://perma.cc/3D3N-2FNY]; Greer Donley, Beatrice A. Chen \& Sonya Borrero, The Legal and Medical Necessity of Abortion Care Amid the COVID-19 Pandemic, 7 J. L. \& BIOSCIENCES 1, 2 (2020).

${ }^{6}$ Donley, Chen, \& Borrero, supra note 6, at 4; Mifeprex (Mifepristone) Information, Food \& Drug Admin., (Apr. 13, 2021), https://www.fda.gov/drugs/postmarket-drug-safety-information-patients-and-providers/mifeprexmifepristone-information [https://perma.cc/3XLE-XRG8].

${ }^{7}$ What is Telehealth?, NEJM CATALYST (Feb. 1, 2018), https://catalyst.nejm.org/doi/full/10.1056/CAT.18.0268 [https://perma.cc/D25M-5G36]; see, e.g., Veronika Williams et al., Using a Mobile Health Application to Support Self-Management in COPD: a Qualitative Study, 64 BRIT. J. OF GEN. PRACTICE e392, e392 (2014).
} 
Professor Farringer's article is optimistic and lays an important framework as we move the delivery of care into the post-pandemic world. Future work will need to be conducted to study and identify the limitations and externalities of telehealth. The framework addresses the limitations vis-à-vis our current healthcare delivery system, but new challenges will arise. It is unclear if opening the door to increased telehealth services will create new underutilization or overutilization issues. New and different fraud and abuse problems may arise. Our current fraud and abuse laws are focused on traditional healthcare delivery. How will these regulations adapt? Of course, not all of these problems can be resolved in one fell swoop. Thus, by providing a framework in which we can implement these policy changes, Professor Farringer's article moves the conversation forward in an important way. Professor Farringer's article is an important contribution to the literature. 